\title{
EVALUACIÓN DE POLICULTIVOS FRUTIHORTÍCOLAS agroecológicos del Nordeste Argentino
}

\author{
EVALUATION OF AGROECOLOGICAL HORTIFFRUIT POLYCULTURES FROM \\ NORTHEASTERn Argentina
}

\author{
Natalia V. Alvez ${ }^{1}$ y Paula Alayón Luaces ${ }^{1 *}$ (D)
}

\begin{abstract}
1. Cátedra de Fruticultura. Facultad de Ciencias Agrarias. Universidad Nacional del Nordeste. Sargento Cabral 2131. (3400). Corrientes, Argentina.

*palayonluaces@yahoo.com
\end{abstract}

Citar este artículo

ALVEZ, N. V. y P. ALAYÓN LUACES. 2020. Evaluación de policultivos frutihortícolas agroecológicos del Nordeste Argentino. Bol. Soc. Argent. Bot. 55: 273-284.

DOI: https://doi. org/10.31055/1851.2372.v55. n2.26899
Recibido: 16 Diciembre 2020

Aceptado: 22 Abril 2020

Publicado: 30 Junio 2020

Editor: Ramiro Aguilar (D)

ISSN versión impresa 0373-580X ISSN versión on-line 1851-2372

\section{SUMMARY}

Background and aims: Agroecology understands that the optimal management of agroecosystems depend on the level of interactions between biotic and abiotic components, and among these interactions the polycultures are key proposals. Within agroecological horticultural systems in northeast Argentina (NEA), the edafoclimatic contributions of fruit trees remains underexplored. Here we evaluate the performance of horticultural crops growing under the canopy of different fruit trees of northeastern Argentina (NEA).

M\&M: Intercropping of four fruit trees, Aguay (Chrysophyllum gonocarpum), Guayabo (Psidium guajava), Ñangapirí (Eugenia uniflora) y Níspero (Eriobotrya japonica), were evaluated in combination with horticultural species: parsley, radish and pea. Available solar radiation determinations for each horticultural species, analysis of litter contribution, weed abundance, and horticultural performance in each fruit tree-horticultural crop combination were measured.

Results: Aguay had a positive effect on the growth and development of the three horticultural species (radish, parsley and peas). The association with Guayabo was positive for parsley but negative for peas. Níspero had a positive effect on pea production; however, the association of Níspero is not recommendable for radish and parsley. The least recommended association for the three horticultural species was with Ñangapirí, for which no positive effects were obtained.

Conclusions: The fruit trees Aguay, Guayabo and Níspero from NEA represent important alternatives for the agroecological production of common horticultural crops such as radish, parsley and peas. In contrast, Ñangapirí fruit tree is not recommended for these horticultural crops.

\section{KEY WORDS}

Agroecology, fruit trees, fruits and vegetables, leaf litter, NEA.

\section{RESUMEN}

Introducción y objetivos: La Agroecología entiende que el manejo óptimo de los agroecosistemas depende del nivel de interacciones entre varios componentes bióticos y abióticos y entre estas, los policultivos son propuestas claves. Aquí evaluamos el comportamiento de especies hortícolas cultivadas debajo de diferentes árboles frutales del nordeste de Argentina (NEA).

M\&M: Se evaluó la consociación de cuatro frutales:Aguay (Chrysophyllum gonocarpum), Guayabo (Psidium guajava), Ñangapirí (Eugenia uniflora) y Níspero (Eriobotrya japonica), en combinación con especies hortícolas: Perejil, Rabanito y Arveja. Se realizaron determinaciones de radiación disponible para las hortícolas, análisis del aporte de la hojarasca, abundancia de vegetación espontánea y rendimiento de las hortícolas en cada combinación.

Resultados: El Aguay tuvo un efecto positivo en el crecimiento y desarrollo de Rabanito, Perejil y Arvejas. La consociación con Guayabo fue positiva para el Perejil pero negativa para las Arvejas. El Níspero tuvo un efecto positivo en la producción de Arvejas; sin embargo la consociación con éste no es recomendable para Rabanito o Perejil. La consociación menos recomendada para las tres especies hortícolas fue con Ñangapirí, en la cual no se obtuvieron efectos positivos.

Conclusiones: Los árboles frutales Aguay, Guayabo y Níspero del NEA representan alternativas importantes para la producción agroecológica de cultivos hortícolas comunes como el Rabanito, el Perejil y las Arvejas. En contraste, el Ñangapirí no se recomienda para estos cultivos hortícolas.

\section{Palabras clave}

Agroecología, frutales, frutihortícolas, hojarasca, NEA. 


\section{INTRODUCCIÓN}

La Agroecología es el estudio holístico de los agroecosistemas, incluidos todos los elementos ambientales y humanos (Altieri, 1995). Centra su atención sobre la forma, la dinámica y función de sus interrelaciones y los procesos en los cuales están envueltas. Entendiendo las relaciones y procesos ecológicos, los agroecosistemas pueden ser manejados para mejorar la producción de forma más sustentable, con menores impactos negativos ambientales y sociales, y un menor uso de insumos externos. Evaluar sistemas agroproductivos, con atención en las características específicas del sistema y en sus interrelaciones, permite entender la importancia de las sinergias para mantener el sistema en equilibrio, en producción constante, sin convertirse en una amenaza para la sostenibilidad (Silva-Laya et al., 2016).

Según Gliessman (2015) la agroecología es una ciencia que busca integrar la ecología con otras disciplinas académicas como la agronomía y la sociología incorporando sistemas de conocimiento local, para orientar la investigación y acciones hacia la transformación sostenible de los sistemas agroalimentarios. Los agroecólogos reconocen que los policultivos, la agroforestería y otros métodos de diversificación imitan los procesos ecológicos naturales y que la sustentabilidad de los agroecosistemas complejos se basa en los modelos ecológicos (León, 2012). Mediante el diseño de sistemas de cultivo que imiten la naturaleza puede hacerse un uso óptimo de la luz solar, de los nutrientes del suelo y de la lluvia (Pret, 1994).

En esencia, el manejo de los agroecosistemas depende del nivel de interacciones entre los varios componentes bióticos y abióticos. A través del ensamble de la biodiversidad funcional es posible iniciar sinergismos que subsidien los procesos del agroecosistema partiendo de proveer servicios ecológicos tales como la activación de la biología del suelo, el reciclado de nutrientes, el aumento de los artrópodos benéficos, los antagonistas de plagas (Altieri \& Nicholls, 1999) y un uso más eficiente del suelo (Gómez Betancur et al., 2018).

En el marco de la biodiversificación de los agroecosistemas, Vandermeer (1989) define a los policultivos como sistemas de cultivo complejos en los cuales dos o más especies son plantadas con una suficiente proximidad espacial que resulta en una competencia o complementación, aumentando, por lo tanto, los rendimientos. Hay evidencia que indica que los sistemas de cultivos diversificados, como los policultivos y la agroforestación son más sustentables y más conservadores de recursos (Vandermeer, 1995). Más aún, los policultivos fueron recientemente denominados por Martin-Guay et al. (2018) como la "nueva revolución verde", ya que tienen el potencial de aumentar la productividad de la tierra explotando las complementariedades de las especies, brindando una oportunidad para lograr una intensificación sostenible de la agricultura. Los resultados de las interacciones en los agroecosistemas están altamente influidos por las condiciones agroecológicas de cada ambiente en particular, de allí la necesidad de evaluar estas interacciones en cada región, atendiendo características específicas que puedan modificar los resultados que surgieran de las combinaciones posibles.

Los árboles frutales nativos son cultivos de tradición y arraigo, que generan sustento para las familias (Brücher, 1989). Según Chifarelli et al. (2019) su utilización en la selva paranaense por los pueblos originarios que la habitaban, se remonta a la coevolución biológica y cultural de los componentes del ecosistema. Estos pueblos utilizaban la fruta fresca que recolectaban en la selva con fines nutricionales desde una perspectiva amplia (física, espiritual y emocional). En los últimos años se incrementó el interés por conocimientos tendientes a mantener y fortalecer la producción frutícola nativa tanto en Argentina (Pintaluba \& Alayón Luaces, 2013) como en otros países americanos, entre ellos México (Lema et al., 2017), Bolivia, Ecuador y Perú (Sanjines Asturizaga et al., 2006; Mostacero León et al., 2017).

El aprovechamiento de los frutales en combinación con hortalizas en contextos familiares y de pequeños productores es una alternativa válida para el acceso a alimentos inocuos y nutritivos para satisfacer sus necesidades alimenticias y poder llevar así una vida activa y sana. El objetivo de este trabajo fue evaluar policultivos de especies hortícolas con frutales leñosos del Nordeste Argentino en un sistema productivo agroecológico.

\section{Materiales y Métodos}

El trabajo se desarrolló en un lote de frutales leñosos no tradicionales que se encuentra ubicado en el Campo Didáctico Experimental de la Facultad 


\section{N. V. Alvez y P. Alayón Luaces - Policultivos frutihortícolas agroecológicos en el NEA}

de Ciencias Agrarias (CDEA) de la Universidad Nacional del Nordeste (UNNE). Ruta Nacional $\mathrm{N}^{\mathrm{o}}$

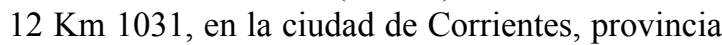
de Corrientes, Argentina.

Las especies de frutales evaluadas fueron: Aguay (Chrysophyllum gonocarpum (Mart, \& Eichler ex Miq.) Engl.; Sapotaceae), Guayabo (Psidium guajava L.; Myrtaceae), Nangapirí (Eugenia uniflora L.; Myrtaceae) y Níspero (Eriobotrya japónica (Thunb.) Lindl.; Rosaceae), en combinación con especies hortícolas de estación otoño-invierno con diferentes órganos de aprovechamiento: hojas de Perejil (Petroselinum crispum Mill. Fuss), tubérculo del hipocótilo de Rabanito (Raphanus sativus L.) y frutos de Arveja (Pisum sativum L.). La importancia de evaluar estas especies de frutales es debido a su adaptación a la región del NEA y a que sus frutos tienen alto potencial de aprovechamiento en la región.

El Aguay o Aguaí dulce es un árbol alto, frondoso, con látex, de hojas obovadas perennes y haz lustroso, de entre 4 a $20 \mathrm{~m}$ de altura y tronco de entre 10 a $70 \mathrm{~cm}$ de diámetro. En Argentina crece en las Provincias Biogeográficas de Las Yungas, entre 300 y 1530 msnm, Chaqueña y Paranense (Salta, Chaco, Formosa, Misiones, Tucumán y Corrientes), extendiéndose a través de los bosques higrófilos de los ríos Paraná y Uruguay hasta el sur de Corrientes y Concepción del Uruguay en Entre Ríos (Tressens, 1996). Entre las propiedades medicinales de sus hojas y semillas se cita su uso para el tratamiento de reumatismos, enfermedades de la piel, sífilis y lepra. Sus frutos son utilizados para la preparación de un dulce tradicional de la región guaranítica (López et al., 2017).

El Guayabo es un árbol de hojas perennes, pero en climas no tropicales se comporta como caducifolio, de hojas simples oblongas o elípticas, el fruto es una baya globosa a ovoide que en el NEA se cosecha desde enero a marzo (Pletsch, 2012), el mismo puede ser utilizado para su consumo en fresco o para fabricar mermeladas, jaleas y jugos. Sus hojas y corteza tienen un largo historial de usos medicinales, se usan para curar la diarrea, disentería, vómitos y dolor de garganta entre otros (Kamath et al., 2008).

El Ñangapirí pertenece a uno de los géneros más abundantes en la familia de las Myrtaceas, con una amplia distribución geográfica en América que va desde México hasta Argentina (Cronquist,
1981). Sus hojas se usan en medicina popular como infusión en el tratamiento de fiebre, reumatismo, enfermedades estomacales, trastornos digestivos, hipertensión y como diurético (Adebajo et al., 1989). La fruta es una baya de 1,5 a $3 \mathrm{~cm}$ de diámetro con ocho surcos longitudinales, es rica en calcio, fósforo, antocianinas, flavonoides, carotenoides y vitaminas $\mathrm{C}$, con un alto poder antioxidante (Melo Silva, 2006).

Si bien el Níspero es una especie nativa del SE de China, la misma fue introducida y se ha naturalizado en la Argentina. En las provincias de Entre Ríos y Misiones es frecuente encontrar ejemplares en selvas marginales degradadas y bordes de selva primaria (Delucchi \& Keller, 2010). Es un árbol o arbusto de 3-10 m de altura con hojas simples, alternas y persistentes. Ampliamente difundido como ornamental y por el aprovechamiento de sus frutos para consumo en frescos, en almíbar, desecados, en confitados, jaleas, mermeladas, dulces, tartas y budines (Valla et al., 1999). También se cita su uso como medicinal ya que las hojas del Níspero se emplean en la medicina hogareña como antitusivo (Amat \&Yajía, 1998), para regular la presión arterial y como tranquilizante (Keller \& Romero, 2006).

Se dividió el lote en 4 parcelas, se seleccionaron al azar 6 árboles frutales adultos en plena producción de cada una de las 4 especies leñosas y se las combinó con la siembra de las 3 especies hortícolas. A modo de control, las tres hortalizas, por su parte, fueron sembradas en el mismo sitio y evaluadas en monocultivo sin la presencia de los árboles frutales.

La siembra se realizó en forma manual, con los cuidados correspondientes y teniendo en cuenta las distancias y época de siembra según el calendario hortícola. Se preparó el suelo con azadas en forma circular, removiendo únicamente el espacio donde se sembraron las especies, los canteros se cubrieron con la hojarasca del frutal. Para la realización de las líneas de siembra se marcaron dos distancias desde los troncos, a $1 \mathrm{~m}$ (circunferencia de 7,60 m lineales) y a vuelo de copa, $1,80 \mathrm{~m}$ distancia (circunferencia 11,50 m lineales). Se utilizó el sistema de chorrillo continuo para Rabanito y Perejil, y para la Arveja sistema de siembra directa. Las semillas no estaban tratadas con ningún tratamiento terapéutico de agroquímicos.

Radiación PAR interceptada por el cultivo: mediante un ceptómetro (barra medidora de 
flujo de fotones, Bar-Rad 100, Cavadevices, Argentina) se realizó la medición de la radiación fotosintéticamente activa (PAR), esto es, la proporción de la radiación solar total que llega a la tierra y es utilizada por las plantas. Las unidades de medida son en flujo de fotones expresadas como $\mu \mathrm{mol} / \mathrm{m}^{2} \mathrm{~s}^{-1}$. Esta medición se realizó a cielo abierto y bajo las copas de los árboles, a una altura media próxima a la copa $(1,30 \mathrm{~m})$ donde se ponderó la luz trasmitida hasta la altura de ramas primarias de la copa y a la altura de las hortalizas $(0,30 \mathrm{~m})$, con el objeto de determinar el ambiente lumínico para las especies hortícolas. La tarea se realizó un día totalmente despejado en el horario del mediodía (12 hs), para obtener el máximo PAR con la menor variación de resultados. La radiación disponible en las diferentes alturas se expresó como porcentaje de la radiación incidente, se consideró como $100 \%$ de PAR el dato a cielo abierto $\left(1550 \mu \mathrm{mol} / \mathrm{m}^{2} \mathrm{~s}^{-1}\right)$.

En la Fig. 1 se representa la distribución de las hortalizas y la incidencia de la luz a diferentes horas del día.

Análisis de suelo: se realizaron análisis de suelo para cada parcela, a partir de una muestra compuesta tomada debajo de cada especie frutal, entre 0-20 $\mathrm{cm}$ de profundidad debajo de la hojarasca. Se determinó materia orgánica en porcentaje $(\mathrm{MO})$ por método de Walkley y Black, $\mathrm{pH}$ con potenciómetro (relación 1:2,5), contenido de nitrógeno $(\mathrm{N})$ método semi-micro Kjeldahl, fósforo $(\mathrm{P})$ por el método Bray Kurtz $\mathrm{N}^{\circ} 1$, potasio $(\mathrm{K})$ por fotometría de llama y calcio $(\mathrm{Ca})$, y magnesio $(\mathrm{Mg})$ por complejometría con EDTA. El suelo del sitio de experimentación ha sido clasificado como Udipsaments álficos, mixta, hipertérmica, perteneciente a la serie Ensenada Grande. Su baja fertilidad natural y susceptibilidad a la erosión, ubica a estos suelos en Subclase II y III (Escobar et al., 1994).

Grado de cobertura de la vegetación espontánea: se estimó el grado de cobertura de las especies herbáceas diferentes a las hortícolas sembradas ("malezas"), utilizando la escala cualitativa propuesta por Ochoa et al. (2012) entendiendo por cobertura a la superficie de suelo que cubre la vegetación espontánea según se detalla en la Tabla 1.

Momento de cosecha: se cuantificaron los días hasta el momento óptimo de cosecha y se compararon con el calendario hortícola para cada cultivo en cada interacción.
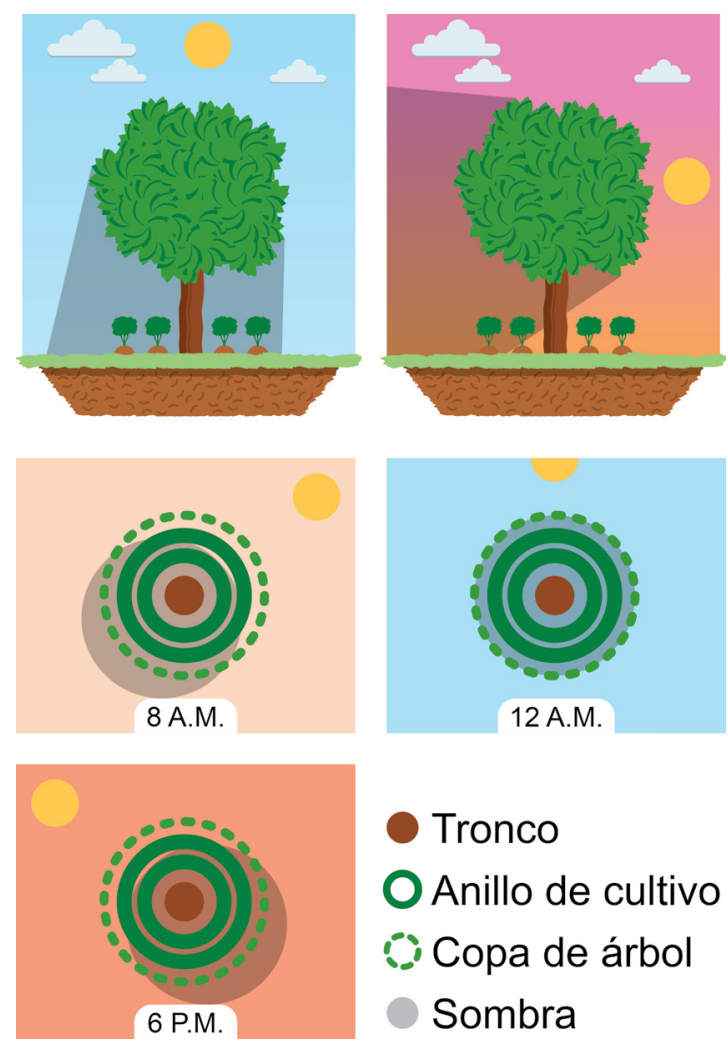

Fig. 1. Detalle de los anillos de las especies hortícolas distribuidas debajo de la copa de los frutales y el sombreamiento a diferentes hora del día.

Rendimiento: al final del ciclo de cada hortaliza, las mismas fueron cosechadas y se evaluaron los rendimientos de los órganos de interés comercial, expresados en $\mathrm{g} / \mathrm{m}^{2}$.

Tabla 1. Escala cuantitativa de cobertura vegetal.

Fuente: Cátedra de Matología, Facultad de Agronomía y Agroindustrias UNSE.

\begin{tabular}{|cc|}
\hline Cobertura & Porcentaje (\%) \\
\hline Ninguna & 0 \\
\hline Pequeña & 1 a 10 \\
\hline Regular & 11 a 20 \\
Mediana Moderada & 21 a 40 \\
Mediana Abundante & 41 a 60 \\
Grande & 61 a 80 \\
Total & 81 a 100 \\
\hline
\end{tabular}




\section{N. V. Alvez y P. Alayón Luaces - Policultivos frutihortícolas agroecológicos en el NEA}

Efecto de la interacción en el crecimiento y desarrollo de las especies hortícolas: se realizó una apreciación cualitativa del crecimiento y desarrollo de las especies hortícolas e incorporando el rendimiento se asignó una estimación siguiendo la siguiente escala:

Muy positivo $(+++)$ : interacción muy favorable, permitió un buen crecimiento y desarrollo de la especie hortícola; rendimiento cercano al $100 \%$ de lo esperado para la especie.

Positivo (++): interacción favorable, permitió el crecimiento y desarrollo de la especie hortícola sin aspectos negativos relevantes, como deformaciones de órganos aprovechables, y con rendimientos mayores al $50 \%$ de lo esperado para cada especie.

Poco positivo $(+)$ : interacción con disminución en crecimiento y desarrollo, y con rendimientos menores al $25 \%$ de lo esperado para la especie.

Negativo (-): interacción desfavorable, las especies hortícolas no prosperaron.

\section{Resultados}

\section{Radiación PAR interceptada por el cultivo}

Se observó que en el Guayabo el ingreso de radiación a nivel de ramas primarias fue del $18,06 \%$, llegando a $25,41 \%$ al nivel de las hortalizas $(7,35 \%$ más; Fig. 2). Diferente fue el caso de las otras tres especies de frutales cuyas copas son más densas que la del Guayabo, por lo cual a nivel de ramas primarias los porcentajes de radiación trasmitida fueron menores y casi sin diferencias a nivel de las hortalizas (Fig. 2). La radiación disponible debajo de la copa del Ñangapirí fue la que presentó valores más bajos, con un porcentaje menor al $2 \%$ del total a la altura de las hortalizas (Fig. 2). El Aguay y el Níspero absorbieron porcentajes de radiación algo menores que el Ñangapirí, con disponibilidad de radiación a nivel de las hortalizas de 2,96\% y 3,15\% respectivamente.

\section{Análisis de suelo}

En todas las muestras de suelo de las especies frutales se observó un $\mathrm{pH}$ ligero a medianamente ácido. En relación a esta variable se destacó el pH con valores más alcalinos de las muestras de Níspero y Ñangapirí y los más ácidos debajo de las plantas de Aguay y Guayabo (Tabla 2). Según Villasanti et al. (2013) los suelos con valores de pH entre 6 y 6,5 son los más adecuados para el desarrollo de la mayoría de las especies hortícolas, siendo las muestras de Ñangapirí y Níspero las que se encontraron en este rango.

Los valores de nutrientes fueron comparados con estándares de referencia de la serie de suelo Ensenada Grande (Escobar et al., 1994; Tabla 2). Se destacan los niveles del aporte de MO de las cuatro especies de frutales, ya que todas se encuentran en el rango de disponibilidad media. Los aportes

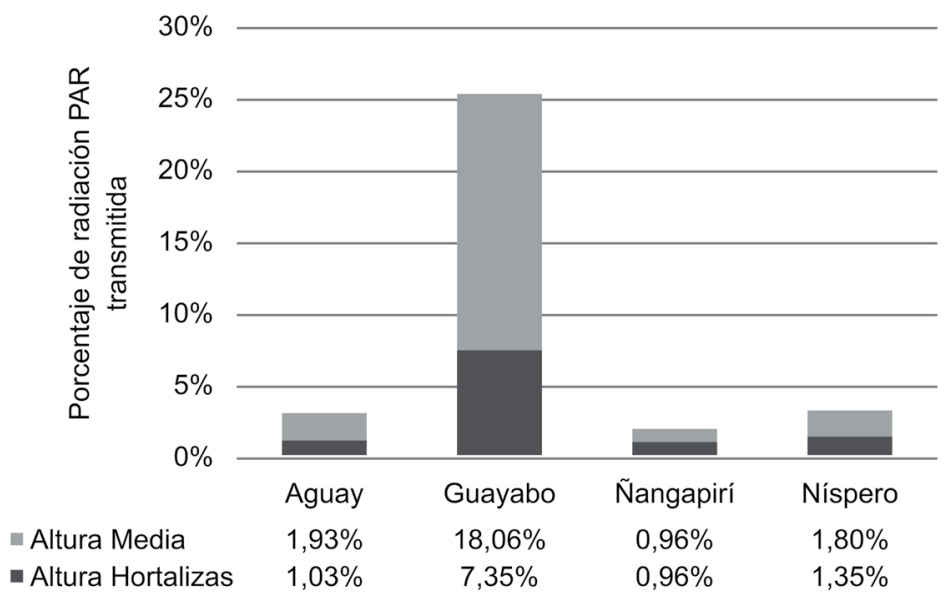

Fig. 2. Radiación fotosintéticamente activa (PAR) que llega a la altura media de la copa $(1,30$ $\mathrm{m})$ y a la altura de las hortalizas $(0,30 \mathrm{~m})$. Representada como porcentaje de lo medido a cielo abierto. 
Tabla 2. Análisis de suelo de la zona de influencia debajo de vuelo de copa de frutales leñosos.

\begin{tabular}{|c|c|c|c|c|c|c|c|}
\hline & $\begin{array}{c}\mathrm{pH} \\
-\end{array}$ & $\begin{array}{c}\text { MO } \\
\%\end{array}$ & $\begin{array}{l}\mathbf{N} \\
\%\end{array}$ & $\begin{array}{c}\mathbf{P} \\
\mathrm{ppm}\end{array}$ & $\begin{array}{c}\mathrm{K} \\
\mathrm{meq} / 100 \mathrm{~g}\end{array}$ & $\begin{array}{c}\mathrm{Ca} \\
\mathrm{meq} / 100 \mathrm{~g}\end{array}$ & $\begin{array}{c}\mathrm{Mg} \\
\mathrm{meq} / 100 \mathrm{~g}\end{array}$ \\
\hline Aguay & 5.65 & 1.44 & 0.13 & 19 & 0.21 & 1.4 & 0.8 \\
\hline Guayabo & 5.59 & 2.51 & 0.24 & 4 & 0.35 & 1.6 & 1.4 \\
\hline Ñangapirí & 6.32 & 1.45 & 0.13 & 6 & 0.17 & 1.8 & 2 \\
\hline Níspero & 6.37 & 2.66 & 0.17 & 4 & 0.23 & 3 & 1 \\
\hline Monocultivo hortalizas & 5.93 & 0.23 & 0.03 & 1 & 0.18 & 0.35 & 1 \\
\hline Serie Ensenada Grande & 7.3 & 0.24 & 0.023 & 13 & 0.14 & $<1$ & $<1$ \\
\hline
\end{tabular}

de MO del Níspero y del Guayabo fueron los más elevados, con un incremento de 10 y 9,45 veces respectivamente, respecto a los valores esperados para la serie de referencia (Tabla 2). Por el contrario, el suelo sin cobertura arbórea presentó valores tan bajos de MO como los de referencia. El contenido de $\mathrm{N}$ también fue más elevado debajo de todos los frutales en relación al de la serie, encontrándose debajo del Guayabo y del Níspero valores más altos de $\mathrm{N}$ que los encontrados debajo del Aguay y del Ñangapirí. El contenido de fósforo del suelo en todas las condiciones debajo de dosel y sin dosel (control) fue muy inferior al observado para la serie de suelo de referencia. El único frutal cuyo aporte presentó valores más elevados que los esperados para la serie fue el Aguay. El contenido de potasio debajo del Aguay, Ñangapirí y Níspero y en condición sin dosel (control) fueron considerados igualmente bajos como la serie de referencia. El aporte del Guayabo se destacó positivamente con más del doble de lo esperado para la serie llegando a valores buenos según escala citada por Saña Vilaseca et al. (1996) (Tabla 2).

Todas las muestras debajo de los árboles presentaron mayor contenido de Ca que el esperado para la serie y el suelo sin dosel; sin embargo, este elemento en todas las mediciones se encuentra deficitario (muy bajo). El contenido de Ca debajo del Níspero, que si bien fue bajo, triplicó lo esperado para la serie (Tabla 2). El contenido de $\mathrm{Mg}$, a diferencia de los otros dos cationes descriptos, presentó niveles altos considerándose en la clase de fertilidad como suelos altamente provistos (Saña Vilaseca et al., 1996), con particularidad las muestras tomadas bajo el Ñangapirí que fue el doble de lo típico de la serie alcanzando el nivel de excesivo.

\section{Grado de cobertura de la vegetación espontánea}

Se observó que bajo el Aguay y el Nangapirí la cobertura de la vegetación espontánea se mantuvo en niveles Regulares (entre 11 y 20\%) mientras que en las condiciones debajo del Guayabo y Níspero, las mismas alcanzaron porcentajes de cobertura Mediana Abundante (entre 41 y 60\%) en combinación con todas las hortalizas (Tabla 3). Por su parte en el monocultivo de Arveja la vegetación espontánea llegó a valores Grandes (entre 61 y 80\%) siendo superior aún en los monocultivos de Perejil y Rabanito, donde alcanzó niveles de cobertura Totales (mayor al 81\%, Tabla 3).

Días y rendimiento de cosecha de hortalizas

Se observó que el cultivo de Perejil presentó muy buenos rendimientos debajo del Aguay y del

Tabla 3. Grado de cobertura de la vegetación espontánea en la zona de influencia debajo de vuelo de copa de frutales leñosos en combinación con especies hortícolas.

\begin{tabular}{|cccccc|}
\hline Interacción & Aguay & Guayabo & Nangapirí & Níspero & Monocultivo hortalizas \\
\hline Perejil & Regular & Mediana Abundante & Regular & Mediana Abundante & Total \\
Rabanito & Regular & Mediana Abundante & Regular & Mediana Abundante & Total \\
Arveja & Regular & Mediana Abundante & Regular & Mediana Abundante & Grande \\
\hline
\end{tabular}




\section{N. V. Alvez y P. Alayón Luaces - Policultivos frutihortícolas agroecológicos en el NEA}

Guayabo, con cosechas de 9,17 y 5,83 veces mayores que en el sistema de monocultivo (Fig. 3). El Rabanito presentó rendimientos más altos en el monocultivo, seguido por el cultivado debajo del Aguay que alcanzó un rendimiento de $380 \mathrm{~g} / \mathrm{m}^{2}$ (Fig. 3). En relación al cultivo de Arvejas, se observó el mayor rendimiento debajo del Níspero, probablemente asociado a los altos contenidos de MO aportados por este frutal. Debajo del Ñangapirí se observaron los rendimientos más bajos para los tres cultivos (Fig. 3).

En la Tabla 4 se presentan las evaluaciones según escala y relacionando el crecimiento, desarrollo y rendimiento de las especies hortícolas con cada combinación de árbol frutal. En esta variable se destaca la interacción con el Aguay. Todas las especies hortícolas debajo del Aguay, tuvieron un rápido crecimiento, buen desarrollo y producción. La gran cobertura del suelo por la hojarasca debajo del frutal no fue un inconveniente para la germinación de las semillas. Debajo de este frutal el crecimiento y desarrollo del Perejil fue muy buena (Fig. Suplem. 1), con un adelanto en la cosecha de entre 10 y 20 días respecto al calendario convencional (Tabla 5). Igual fue el comportamiento del Rabanito, cuyos tubérculos no presentaron deformaciones ni alteraciones en su desarrollo (Fig. Suplem. 2) probablemente muy beneficiados por el aporte nutricional, en particular el $\mathrm{P}$ de la hojarasca del Aguay. Las Arvejas llegaron a cosecha en igual tiempo que el esperado en sistemas convencionales presentando un buen desarrollo y estado general en esta combinación (Fig. Suplem. 3).

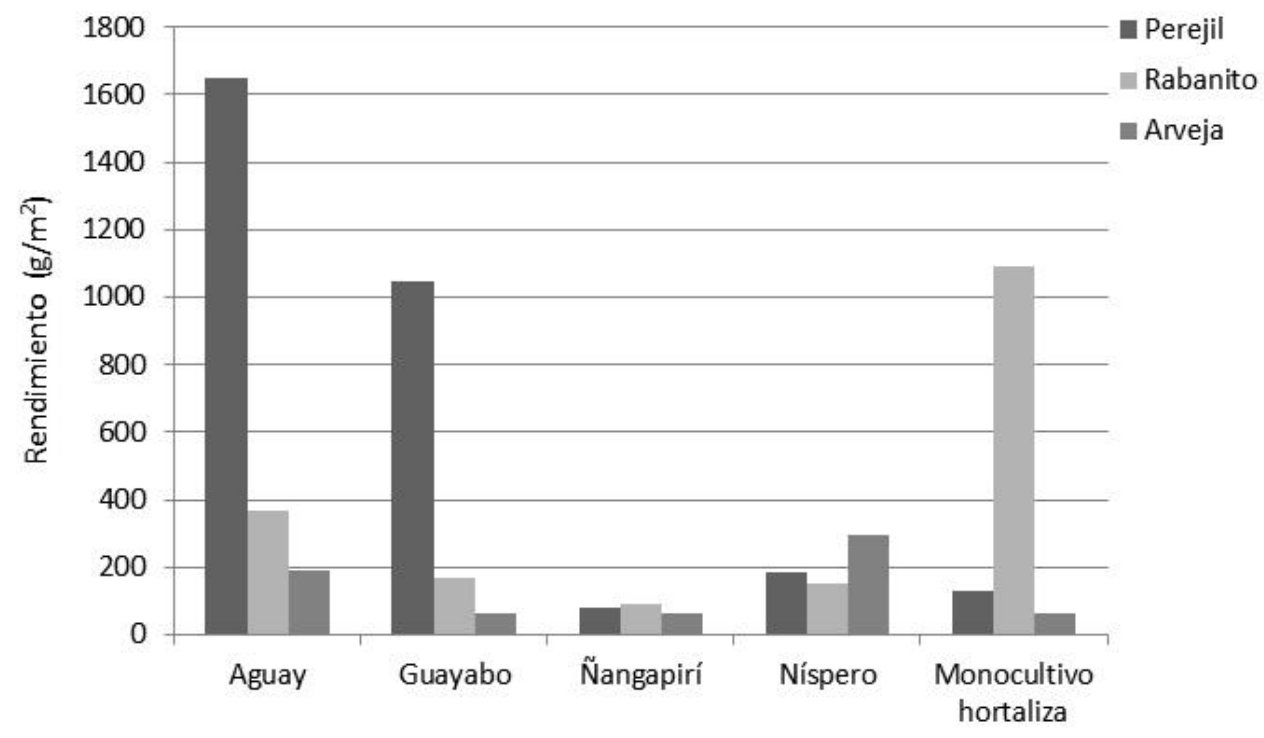

Fig. 3. Rendimiento de las hortalizas en cada combinación con frutales y en monocultivo (en g/ $\left.\mathrm{m}^{2}\right)$.

Tabla 4. Efecto de las interacciones de frutales leñosos en el crecimiento y desarrollo de especies hortícolas.

\begin{tabular}{|cccccc|}
\hline Interacción & Aguay & Guayabo & Nangapirí & Níspero & Monocultivo hortalizas \\
Perejil & +++ & ++ & - & + & - \\
Rabanito & ++ & + & - & + & +++ \\
Arveja & ++ & + & - & ++ & - \\
\hline
\end{tabular}


Tabla 5. Días para el momento óptimo de cosecha de las especies hortícolas.

\begin{tabular}{|ccccccc|}
\hline Interacción & Aguay & Guayabo & Nangapirí & Níspero & $\begin{array}{c}\text { Monocultivo } \\
\text { hortalizas }\end{array}$ & $\begin{array}{c}\text { Calendario } \\
\text { hortícola }\end{array}$ \\
Perejil & 90 & 90 & 150 & 90 & 150 & $100-110$ \\
Rabanito & 45 & 45 & 120 & 45 & 35 & $35-40$ \\
Arveja & 100 & 100 & 120 & 90 & 100 & $100-110$ \\
\hline
\end{tabular}

Las especies hortícolas debajo del Guayabo que tuvieron mejor comportamiento fueron el Perejil y las Arvejas respecto al Rabanito, aunque todas más limitadas en comparación con el Aguay. En el desarrollo del Rabanito en esta combinación se observaron tubérculos de distintos tamaños y con deformidades a los 45 días de sembrados (Fig. Suplem. 4) esta respuesta probablemente fue provocada por la acción física de las raíces del frutal que están dispuestas en un sistema superficial y concentradas en las primeras capas del suelo, de 0 a $30-40 \mathrm{~cm}$ (Bandera Fernández \& Pérez Pelea, 2015).

En las combinaciones con Níspero se observó un rápido crecimiento $\mathrm{y}$ desarrollo muy bueno principalmente de las Arvejas, que ya a los 90 días se encontraban en el momento óptimo para la cosecha con los rendimientos más elevados de las combinaciones evaluadas (Fig. 3).

La interacción del Ñangapirí sobre las especies hortícolas se caracterizó por ser limitante a negativa. Esta especie fue, de las cuatro evaluadas, la que mayor radiación PAR interceptó (Fig. 2), por lo que las especies hortícolas debajo de este frutal se encontraban en condiciones de mayor intensidad de sombra, lo cual pudo haber sido uno de los factores que comprometió el crecimiento y rendimiento general de las mismas. A vuelo de copa de este frutal, el desarrollo de todas las especies hortícolas fue irregular y de crecimiento lento (Fig. Suplem. 5), lo cual además incidió en que presentaran ciclos de cosecha más largo (Tabla 4).

\section{Discusión}

La radiación fotosintéticamente activa (PAR) captada por un vegetal determina directamente la producción de fotosintatos, influyendo sobre el crecimiento, la productividad y calidad de fruta de las plantas (Ferree, 1980). Las variaciones temporales en la intensidad de la radiación solar tienen consecuencias ecológicas sobre las plantas afectando su fotosíntesis, morfología, tolerancia a la sombra, crecimiento y supervivencia (Kimmins, 1987). Se ha demostrado que las modificaciones de la estructura de la copa, forma de conducción y del tamaño del árbol frutal inciden en la intercepción y distribución de la luz (Heinicke, 1964; Robinson, et al., 1991; Raffo \& Iglesias, 2004). Las diferentes arquitecturas de las especies frutales evaluadas, incidieron en la disponibilidad de luz para las hortalizas cultivadas bajo sus copas, lo cual coincide con lo reportado por Robinson (2011) quien manifiesta que la intercepción de luz es en gran medida una función de la forma y la disposición del árbol. La copa abierta que conforman las ramas del Guayabo facilitó la entrada de luz a través del canopeo, mientras que el Aguay, el Níspero y el Ñangapirí tienen copas más cerradas, por lo que interceptaron más radiación. Sin embargo esta mayor intercepción de luz, en el caso del Aguay y del Níspero, no incidió negativamente en la producción de las especies hortícolas.

La fertilidad del suelo se refiere a su capacidad para dar soporte y manutención al desarrollo y crecimiento de las plantas, lo cual se relaciona con la disponibilidad de nutrientes esenciales para su absorción. Esto se ve facilitado por sus reservas en la materia orgánica del suelo, el reciclaje de nutrientes de las formas orgánicas a minerales disponibles para las plantas y los procesos fisicoquímicos que controlan su fijación y liberación (Srivastava, 2013; Srivastava \& Malhotra, 2014). De allí la importancia del aporte de MO de la hojarasca de especies perennes, observándose en este trabajo que todas las especies frutales estudiadas mejoraron este parámetro debajo de los vuelos de copa, en particular se destacan los elevados aportes del Guayabo y el Níspero. En consecuencia, también los contenidos de nutrientes $(\mathrm{N}, \mathrm{P}, \mathrm{K}, \mathrm{Mg}$ y $\mathrm{Ca}$ ) provenientes de 


\section{N. V. Alvez y P. Alayón Luaces - Policultivos frutihortícolas agroecológicos en el NEA}

la hojarasca fueron superiores a los de la serie lo cual generó un sitio apropiado para la nutrición de los cultivos hortícolas evaluados. El P es un nutriente clave para aumentar la productividad de las legumbres y cuando su nivel es bajo representa el factor más importante responsable de la baja productividad de las mismas (Sarawgi et al., 2005). El aporte de la hojarasca del Aguay incrementó muy positivamente la concentración de $\mathrm{P}$, con beneficios para el desarrollo de las especies hortícolas.

La vegetación espontánea o adventicia, es toda aquella vegetación que no es cultivo y que se encuentra presente en los agroecosistemas, tanto dentro como fuera de las parcelas de cultivo (Vicente \& Sarandón, 2013). Se observó que en los cuatro tipos de policultivos disminuyó la cobertura de la vegetación espontánea creciendo junto a las hortalizas en comparación con la situación de monocultivo, que presentó una muy elevada cobertura de vegetación espontánea no deseada. Esta disminución, consolida lo propuesto por Altieri (1994) quien afirma que los policultivos tienen efectos positivos pues disminuyen el crecimiento de especies herbáceas que compiten con los cultivos, lo que implica un menor uso de mano de obra, menos productos químicos y por lo tanto de costos. En las interacciones con estos frutales, la menor presencia de vegetación espontánea encontrada debajo del Aguay se asoció con la hojarasca que dejó esta especie, aunque no sería un efecto de la cantidad de la hojarasca, ya que la del Aguay fue menor que la encontrada debajo de Guayabo y Níspero que presentaron mayores coberturas de vegetación espontánea (Tabla 3). Por lo que la menor presencia de vegetación espontánea debajo del Aguay podría deberse a que su hojarasca contenga algún compuesto que ejerza un efecto alelopático que afecte negativamente el crecimiento de la misma. Por su parte, la restricción del desarrollo de vegetación espontánea en relación a la interacción con el Ñangapirí, estaría asociada a los efectos alelopáticos conocidos para el género (Souza et al., 2007; Giotto et al., 2007; Pina et al., 2009) y al intenso sombreamiento que imparte la especie.

El comportamiento de las tres especies hortícolas evaluadas en consociación con el Aguay fue positivo, este frutal aporta nutrientes y condiciones adecuadas para el desarrollo de las mismas con buenos rendimientos y apropiados periodos de tiempos de los cultivos hasta la cosecha. En las interacciones con el Guayabo se destacó el desarrollo y rendimiento del Perejil, el aporte de MO y consecuentemente de N, favoreció el desarrollo del órgano de aprovechamiento de esta especie ya que la disponibilidad de $\mathrm{N}$ incide directamente en el desarrollo de biomasa (Chenard et al., 2005). La consociación con Níspero favoreció el cultivo de Arveja, la particularidad de esta hortaliza es que se desarrolla mejor en suelos con $\mathrm{pH}$ alcalinos o levemente ácidos, y en relación a este parámetro el aporte de la hojarasca del Níspero fue el que confirió el valor de $\mathrm{pH}$ más elevado de los cuatro frutales evaluados. La cantidad de hojarasca presente debajo del frutal limitó el crecimiento óptimo del Rabanito y Perejil luego de la emergencia.

A diferencia de las otras tres especies frutales evaluadas se observó un comportamiento poco favorable para las especies hortícolas asociadas al Ñanagapirí, estas observaciones podrían ser asociadas a las encontradas en otra especie del mismo género (Eugenia dysenterica), en donde Souza et al. (2007); Giotto et al. (2007) y Pina et al. (2009), observaron efectos alelopáticos negativos sobre el desarrollo de Sesamum indicum, Raphanus sativus y Lactuca sativa.

\section{Conclusiones}

De las observaciones realizadas se concluye que los frutales leñosos del Nordeste Argentino son una alternativa importante a considerar para la producción de policultivos agroecológicos en asociación con hortalizas, con diferentes niveles de respuestas según el frutal y la especie hortícola. Se observaron incidencias variables en las consociaciones estudiadas, siendo la más recomendable aquellas con Aguay (Chrysophyllum gonocarpum) y la menos recomendable aquellas con Ñangapirí (Eugenia uniflora).

\section{Contribución DE LOS AUtORES}

Ambas autoras diseñaron y realizaron la investigación conjuntamente, también su interpretación y redacción del manuscrito a partes iguales. 


\section{Agradecimientos}

A la Secretaría General de Ciencia y Técnica de la Universidad Nacional del Nordeste por el financiamiento para la realización del trabajo.

\section{Biblografía}

ADEBAJO, A.C., K.J. OLOKI \& A. ALADESANMI. 1989. Antimicrobial activity of the leaf extract of Eugenia uniflora. J. Phytotherapy Resource 3:258259. https://doi.org/10.1002/ptr.2650030608

ALTIERI, M.A. 1994. Agroecología: principios y estrategias para diseñar una agricultura que conserva recursos naturales y asegura la soberanía alimentaria. Disponible en: http://ambiental. uaslp.mx/Agricultura/2002\%201-Altieri\%20 Agroecologia_principios_y_estrategias.pdf[Acceso: 17October 2019].

ALTIERI, M.A.1995. Agroecology: the science of sustainable agriculture. Westview Press, Boulder, CO.

ALTIERI, M.A. \& C.I. NICHOLLS. 1999. Biodiversity, ecosystem function, and insect pest management in agricultural systems. In: COLLINS, W.W. \& C.O. QUALSET (eds.), Biodiversity in Agroecosystems, pp. 69-84.CRC Press.

AMAT, A.G. \& M.E. YAJÍA. 1998. Plantas populares utilizadas en la fitoterapia popular de la provincia de Misiones (Argentina). In: A.G. AMAT (ed.): Farmacobotánica y Farmacognosia en Argentina, 1980-1998. pp. 205-230. Ediciones Científicas Americanas, La Plata.

BANDERA FERNÁNDEZ, E. \& L. PÉREZ PELEA. 2015. Revisión Bibliográfica: mejoramiento genético de guayabo (Psidium guajava L.). Cultivos Tropicales 36: 96-110.

BRÜCHER, H. 1989. Aromatic and Fleshy Fruits. In: Useful Plants of Neotropical Origin. Springer, Berlin, Heidelberg. https://doi.org/10.1007/978-3-642-73313-0_11

CHENARD, C.H., D.A. KOPSELL \& D.E. KOPSELL. 2005. Nitrogen concentration affects nutrient and carotenoid accumulation in parsley. J. Plant Nutr. 28: 285-297. https://doi.org/10.1081/PLN-200047616

CHIFARELLI, D., E. DESCALZI, L. BRUSCA \& C. GELABERT. 2019. Sistemas agroforestales y frutales nativos. Estrategia de producción y conservación desde la agricultura familiar. Leisa Revista de agroecología 35: 27-30
CRONQUIST, A. 1981. An integrated system of classification of flowering plants. New York: Columbia University Press.

DELUCCHI, G. \& H.A. KELLER. 2010. La naturalización del «níspero», Eriobotrya japonica en la Argentina. Bonplandia 19: 71-77. https://doi.org/10.30972/bon.1911335.

ESCOBAR, E.H, D. LIGIER, R. MELGAR, H. MATTEIO \& O. VALLEJOS. 1994. Mapa de Suelo de los Departamentos Capital, San Cosme e Itatí, de la Provincia de Corrientes. INTA-CFI-ICA.

FERREE, D.C. 1980. Canopy development and yield efficiency of Golden Delicious apple trees in four orchard management systems. J. Amer. Soc. Hort. Sci. 105: 376-380.

GIOTTO, A.C., S.C.C. OLIVEIRA \& J.G.P. SILVA. 2007. Efeito alelopático de Eugenia dysenterica Mart. ex DC. Berg. (Myrtaceae) nagerminação e no crescimento de Lactuca sativa L. (Asteraceae). Rev. Bras. Biocienc. 5: 600-602. https://doi.org/10.26512/2016.03.d.21109

GLIESSMAN, S.R. 2015. Agroecology: the ecology of sustainable food systems. Boca Raton, FL: CRC Press. Taylor \& Francis.

GÓMEZ BETANCUR, L.M., S.M. MÁRQUEZ GIRÓN \& L.F. RESTREPO BETANCUR. 2018. La milpa como alternativa de conversión agroecológica de sistemas agrícolas convencionales de frijol (Phaseolus vulgaris), en el municipio El Carmen de Viboral, Colombia. Idesia (Arica) 36: 123-131.

HEINICKE, D.R. 1964. The micro-climate of fruit trees. III. The effect of tree size on light penetration and leaf area in 'Red Delicious' apple trees. Proc. Amer. Soc. Hort. Sci.85:33-41.

KAMATH, J.V., C.K. NAIR RAHUL, S. ASHOK KUMAR \& S. MOHANA LAKSHMI. 2008. Psidium guajava L: A review. Int. J. Green Pharmacy. 2: 9-12.2. https://doi.org/10.4103/0973-8258.39155

KELLER, H.A. \& H.F. ROMERO. 2006. Plantas medicinales utilizadas por campesinos del área de influencia de la Reserva de Biósfera Yabotí (Misiones, Argentina). Bonplandia 15:125-141. https://doi.org/10.30972/bon.153-4103

KIMMINS, J.P. 1987. Forest ecology: A foundation for sustainable management (2a ed.). New Jersey, USA: Prentice Hall.

LEMA, F.P., O.M.A. PÉREZ, D.M.T. MARTÍNEZ \& G.H. NAVARRO. 2017. Importancia y caracterización de frutales criollos en dos 


\section{N. V. Alvez y P. Alayón Luaces - Policultivos frutihortícolas agroecológicos en el NEA}

comunidades de Tuxpan, Veracruz. Rev. Colomb. Cienc. Hortic. 11: 306-313.

https://doi.org/10.17584/rcch.2017v11i2.7590

LEÓN, T. 2012. Agroecología: La ciencia de los agroecosistemas - la perspectiva ambiental. IDEA, Universidad Nacional de Colombia, Bogotá.

LÓPEZ, A.E, P. ALAYÓN LUACES \& D. TAIARIOL. 2017. Aguay: Chrysophyllum gonocarpum. PROCISUR. IICA. $18 \mathrm{pp}$.

MARTIN-GUAY, M.O., A. PAQUETTE, J. DUPRAS \& D. RIVEST. 2018. The new green revolution: sustainable intensification of agriculture by intercropping. Sci. Total Environ. 615: 767-772. https://doi.org/10.1016/j.scitotenv.2017.10.024

MELO SILVA, S. 2006. Pintanga. Rev. Bras. Frutic. 28: 1-159.

MOSTACERO LEÓN, J., F. MEJÍA COICO, D. GASTAÑADUI ROSAS, J. DE LA CRUZ CASTILLO. 2017. Inventario taxonómico, fitogreográfico y etnobotánico de frutales nativos del norte del Perú. Scientia Agropecuaria 8: 215-224. https://doi.org/10.17268/sci.agropecu.2017.03.04

OCHOA, M.C., M.T. SOBRERO \& S. CHAILA. 2012. Integración de conocimientos teóricos y prácticos mediante métodos de relevamiento y evaluación de malezas en una práctica a campo. Cátedra de Matología, Facultad de Agronomía y Agroindustrias UNSE. Disponible en: http://sedici. unlp.edu.ar/bitstream/handle/10915/21760/519In t e g r a c i o n $+\mathrm{de}+\mathrm{conocimientos}$. pdf?sequence=1[Acceso: 17October 2019].

PINA, G.O., F. BORGHETTI, C.E.S. SILVEIRA \& L.A.R. PEREIRA. 2009. Effects of Eugenia dysenterica leaf extracts on the growth of sesame and radish. Allelopathy J. 23: 313-322.

PINTALUBA, N. \& P. ALAYÓN LUACES. 2013. Caracterización de frutas comestibles de especies nativas de uso popular en el Parque Provincial "Salto Encantado Del Valle Del Cuñá Pirú - Misiones. Bonplandia 22: 79-89.

https://doi.org/10.30972/bon.2221249

PLETSCH, R. 2012. Calendario Frutícola para la Provincia de Corrientes Agencia de Extensión rural Corrientes. Disponible en: https://inta.gob.ar/sites/default/files/ script-tmp-inta-_calendario_frutcola_final2red.pdf

PRET, J.N.1994. Regenerating agriculture. Earth scan Publications Ltd., London.

RAFFO, M.D. \& N. IGLESIAS. 2004. Efecto de la intercepción y distribución de la radiación fotosintéticamente activa en manzanos cv. Fuji, bajo cuatro sistemas de conducción en alta densidad. Revista RIA 33: 29-42.

https://doi.org/10.12706/itea.2017.002

ROBINSON, T., A. LAKSO \& Z. REN. 1991. Modifying Apple Tree Canopies for Improved Production Efficiency. Hort. Science 26: 1005-1012.

https://doi.org/10.21273/hortsci.26.8.1005

ROBINSON, T. 2011. Advances In Apple Culture Worldwide. Rev. Bras. Frutic. Volumen Especial, E: $37-47$.

SANJINES ASTURIZAGA, A., B. ØLLGAARD \& H. BALSLEV. 2006. Frutos comestibles. Botánica Económica de los Andes Centrales, Universidad Mayor de San Andrés, La Paz, 329-346.

SAÑA VILASECA, J., J.C. MORÉ RAMOS \& A. COHÍ RAMÓN. 1996. La gestión de la fertilidad de los suelos: fundamentos para la interpretación de los análisis de suelos y la recomendación de abonado. Ministerio de Agricultura, Pesca y Alimentación. España.

SARAWGI, S.K., A.P. SINGH \& K.K. PUROHIT. 2005. Effect of phosphorus on nodulation, uptake and economics of soyabean varieties in vertisols. Ann. Plant Soil Res. 7: 165-168.

SILVA-LAYA, S.J., PÉREZ-MARTÍNEZ, S. RÍOSOSORIO, L.A. 2016. Evaluación agroecológica de sistemas hortícolas de dos zonas del oriente antioqueño, Colombia. Revista Colombiana de Ciencias Hortícolas 10: 355-366.

http://dx.doi.org/10.17584/rcch.2016v10i2.5752

SOUZA, L.M.D., G.B. CANINI, S.S. AIRES \& F. BORGHETTI. 2007. Efeito alelopático de folhas de quatro espécies do cerrado sobre crescimento de gergelim. Rev. Bras. Biocienc. 5: 540-542.

SRIVASTAVA, A.K. 2013. Site specific nutrient management in citrus. Scientific Journal of Agriculture 2: 1-15.

SRIVASTAVA, A.K. \& S.K. MALHOTRA. 2014. Nutrient management in fruit crops: Issues and strategies. Indian Journal Fertilizer 10:72-88.

TRESSENS, S.G. 1996. Sapotaceae. Flora Fanerogámica Argentina. Fascículo 30: 1-9 1999. En: F. ZULOAGA. \& O. MORRONE (eds.). Catálogo de las Plantas Vasculares de la República Argentina II. Missouri Botanical Garden 10271029.

VALLA, J.J., L. JANKOWSKI, D. BAZZANO \& A.J. HERNÁNDEZ. 1999. Árboles Urbanos. En: H.B. LAHITTE \& J.A. HURRELL (eds.). Biota Rioplatense 4. LOLA, Buenos Aires. 
Bol. Soc. Argent. Bot. 55 (2) 2020

VANDERMEER, J. 1989. The ecology of intercropping. Cambridge University Press.

VANDERMEER, J. 1995. The ecological basis of alternative agriculture. Annu. Rev. Ecol. Syst. 26: 201-224. https://doi.org/10.1146/annurev.es.26.110195.001221

VICENTE, L.A. \& S.J. SARANDÓN. 2013. Conocimiento y valoración de la vegetación espontánea por agricultores hortícolas de La Plata,
Argentina. Su importancia para la conservación de la agrobiodiversidad Rev. Bras. de Agroecologia. 8: 57-71.

VILLASANTI, C., P. ROMÁN \& A. PANTOJA. FAO. 2013. El manejo del suelo en la producción de hortalizas con Buenas Prácticas Agrícolas. Disponible en: http://www.fao.org/3/a-i3361s. pdf[Acceso: 17October 2019]. 
N. V. Alvez y P. Alayón Luaces - Policultivos frutihortícolas agroecológicos en el NEA

\section{Figuras Suplementarias}

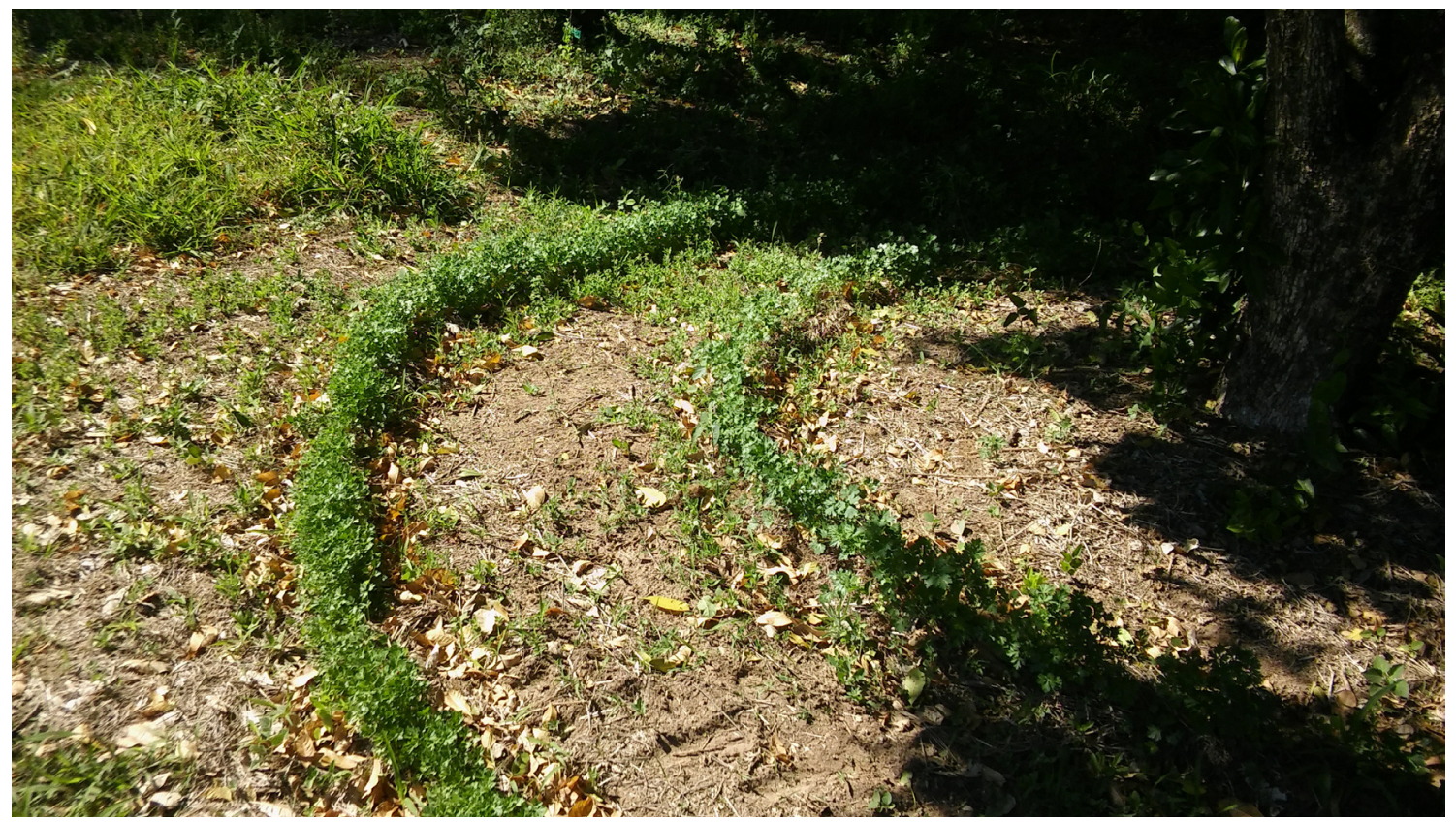

Fig. S1. Cultivo de Perejil (Petroselinum crispum) debajo de Aguay (Chrysophyllum gonocarpum).

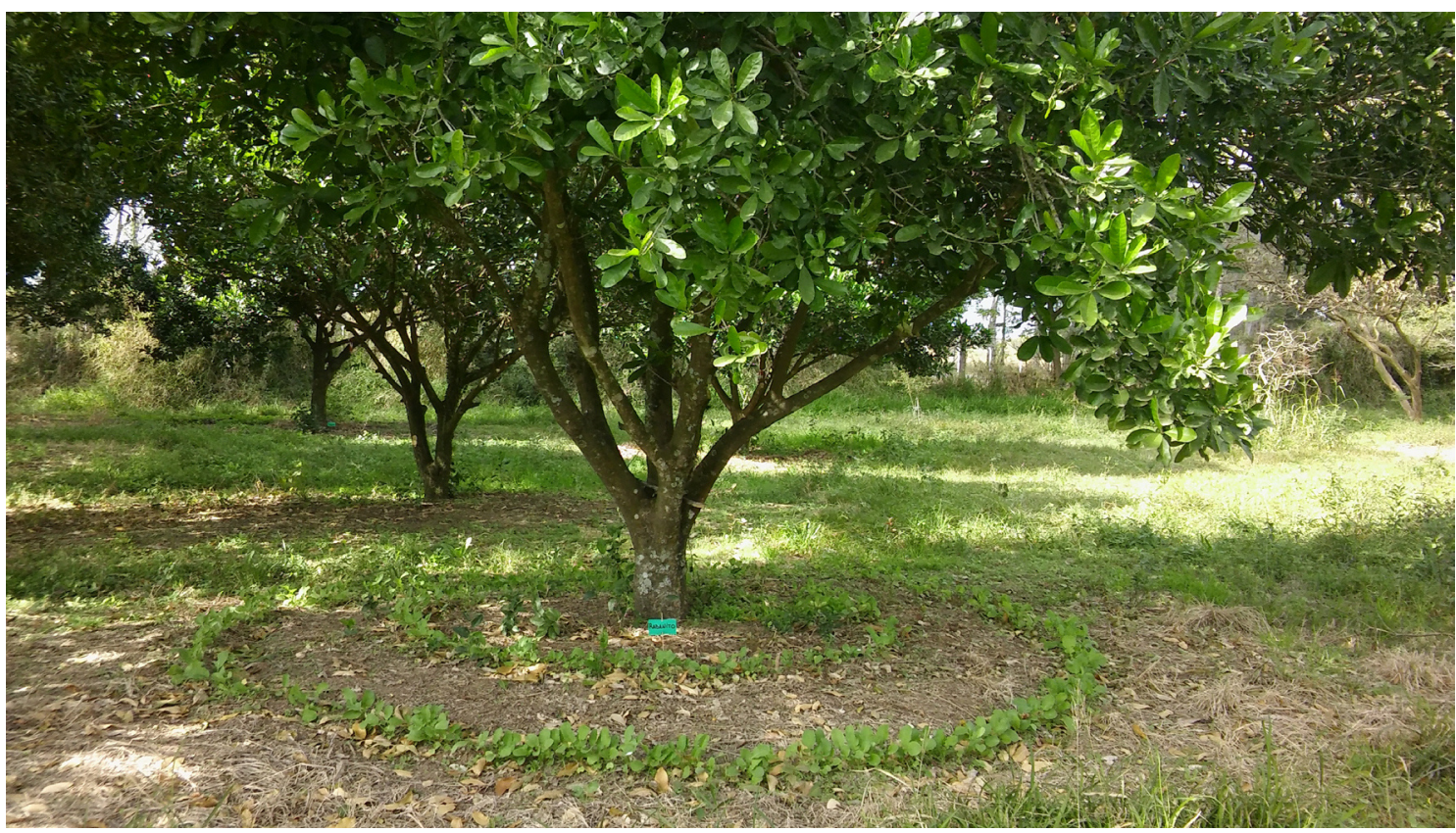

Fig. S2. Cultivo de Rabanito (Raphanus sativus) debajo de Aguay (Chrysophyllum gonocarpum). 
Bol. Soc. Argent. Bot. 55 (2) 2020

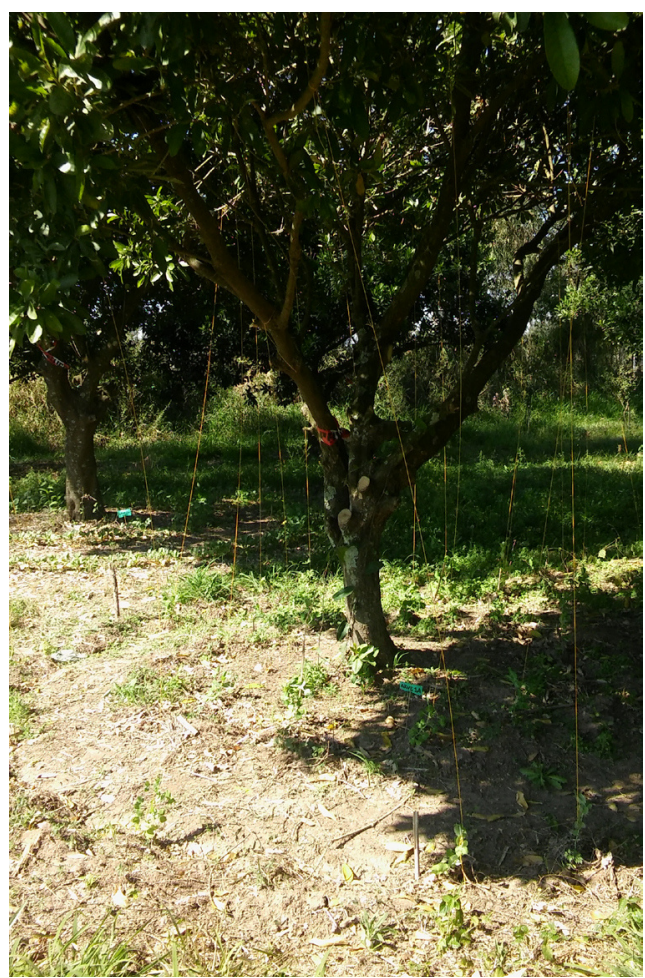

Fig. S3. Cultivo de Arveja (Pisum sativum) debajo de Aguay (Chrysophyllum gonocarpum).

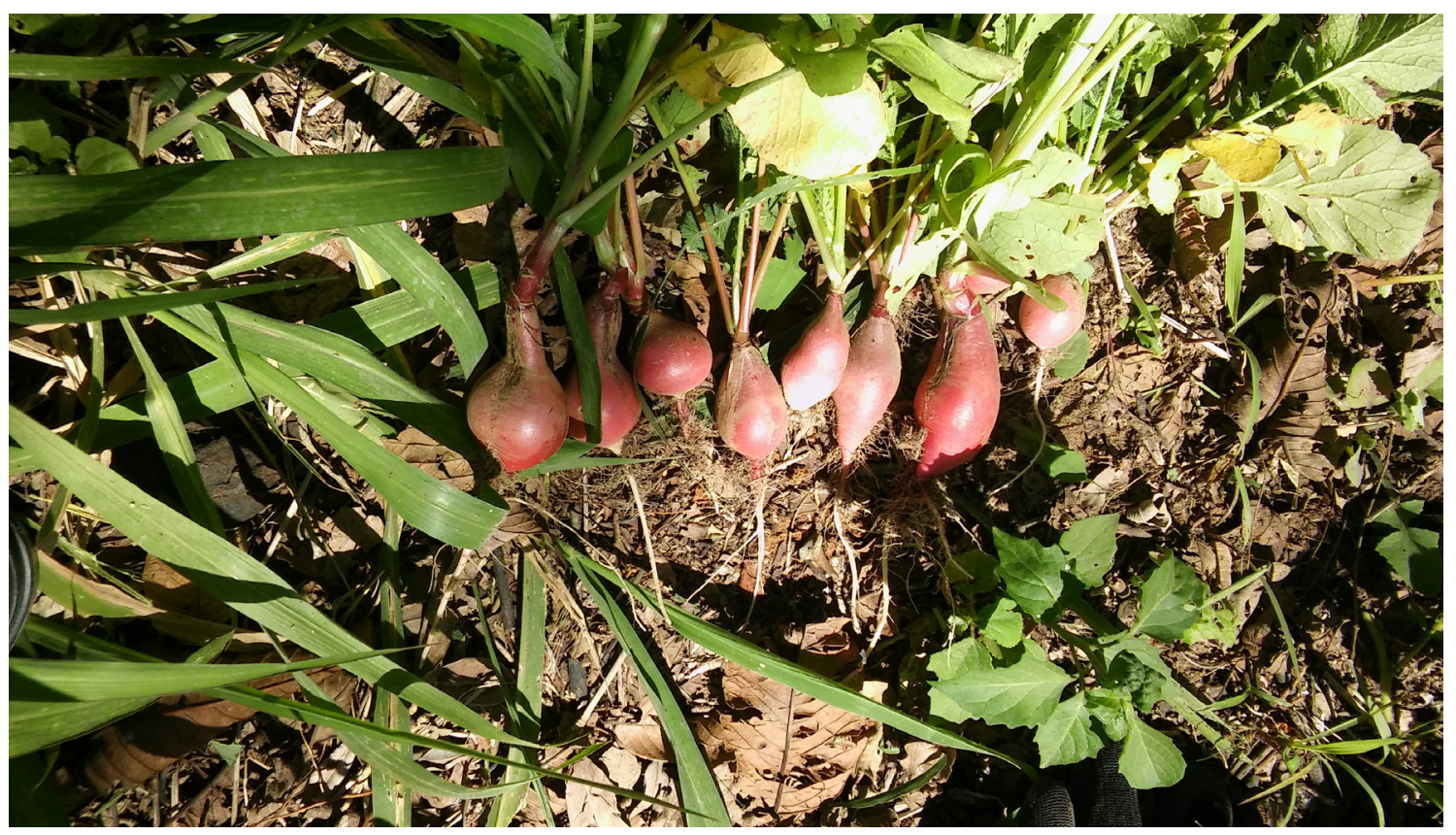

Fig. S4. Cultivo de Rabanito (Raphanus sativus) debajo de Guayabo (Psidium guajava) se observa tubérculos deformados provenientes de esta combinación de cultivos 
N. V. Alvez y P. Alayón Luaces - Policultivos frutihortícolas agroecológicos en el NEA

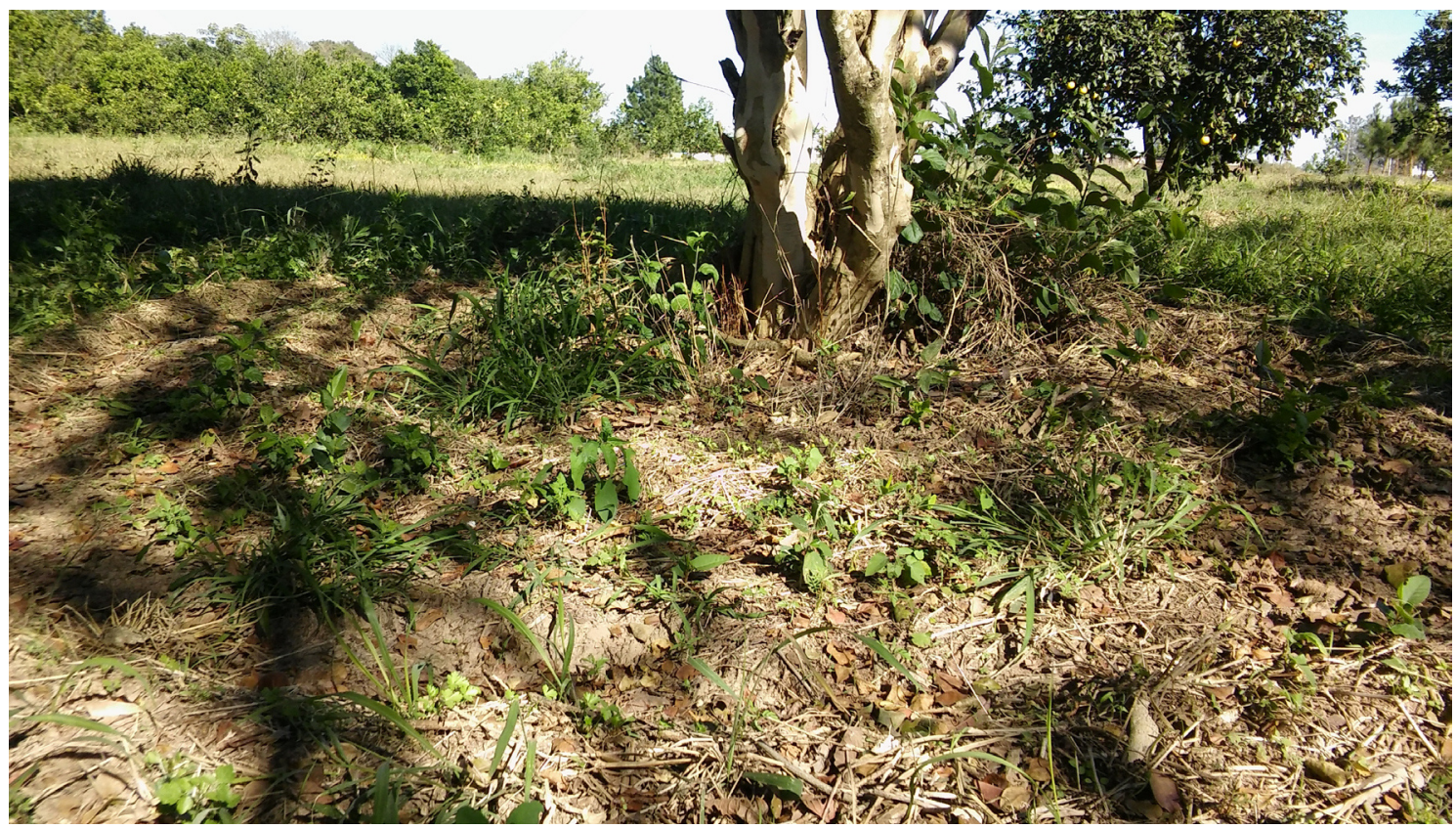

Fig. S5. Cultivo de Perejil (Petroselinum crispum) debajo de Ñangapirí (Eugenia uniflora) con muy poco desarrollo y crecimiento de la hortaliza. 